\title{
Pratiques
}

Linguistique, littérature, didactique

141-142| 2009

La synonymie

\section{De la synonymie, vue à travers les emplois des mots synonyme, synonymie et synonymique dans les textes}

Michelle Lecolle

\section{(2) OpenEdition}

\section{Journals}

Édition électronique

URL : https://journals.openedition.org/pratiques/1317

DOI : 10.4000/pratiques.1317

ISSN : 2425-2042

Éditeur

Centre de recherche sur les médiations (CREM)

Édition imprimée

Date de publication : 15 juin 2009

Pagination : 121-137

Référence électronique

Michelle Lecolle, «De la synonymie, vue à travers les emplois des mots synonyme, synonymie et synonymique dans les textes », Pratiques [En ligne], 141-142 | 2009, mis en ligne le 20 juin 2014, consulté le 10 mars 2023. URL : http://journals.openedition.org/pratiques/1317 ; DOI : https://doi.org/ 10.4000/pratiques. 1317

Tous droits réservés 


\title{
De la synonymie, vue à travers les emplois des mots synonyme, synonymie et synonymique dans les textes
}

\author{
Michelle Lecolle
}

CELTED, Université Paul-Verlaine, Metz

Le présent travail se propose d'aborder la synonymie par le biais de l'emploi de synonyme (singulier et pluriel) et de ses dérivés dans des textes.

Le choix d'une entrée sémasiologique (par le mot) amène à un déplacement de l'objet d'étude : de la notion de synonymie aux emplois et aux valeurs des mots correspondants. Le premier objectif de l'article est donc d'examiner les conséquences de ce déplacement : en deux mots, que reste-t-il de la notion de synonymie - en tant que relation paradigmatique sémantique lexicale - dans l'emploi de ces mots? Quelles valeurs, similaires ou différentes, peut-on y repérer? Quel sens, en somme, est conféré au mot synonyme et à ses dérivés?

L'approche proposée est discursive : c'est dans des énoncés, des fragments de textes, en co(n)texte donc, que sont analysés les emplois des mots métalinguistiques synonyme et dérivés. Supposant que ces énoncés portent une trace du sentiment linguistique sur l'usage de ces mots et sur leur sens, je postule en outre que l'emploi de synonyme et alii est moins anodin que celui de mots ou expressions également métalinguistiques tels que signifier, sens de ${ }^{(1)}$, etc. Ces derniers sont en effet plus polysémiques, et paraissent davantage se fondre dans un usage ordinaire varié, s'appliquant d'ailleurs souvent à tout autre chose qu'à du sens proprement lexical. Ce postulat de départ est au fondement de la constitution de mon corpus et de certaines des observations qui en découlent.

De fait, l'observation des emplois de synonyme et, dans une moindre mesure, de ses dérivés, fait émerger des valeurs, récurrentes mais plurielles, de ces mots, et peut-être de la notion de synonymie. En effet, alors que, pour le linguiste, la synonymie est une relation d'équivalence ou de proximité de sens entre des unités lexicales, les emplois de synonyme s'éloignent de cette valeur. Ainsi, dans une phase préparatoire au présent travail, j'ai rencontré, en nombre significatif, et dans divers discours, des énoncés du type de (1) :

(1) Voir Julia (2001) sur l'emploi de sens dans les gloses de spécification du sens. La perspective adoptée ici est proche de celle de cet ouvrage. 
(1) «Je comprends la volonté de gérer l'immigration, et de protéger les intérêts des français, mais ce ne doit pas être synonyme de discrimination automatique pour les non occidentaux. " (internaute, sur le site Les amoureux au ban public ${ }^{(2)}$ ),

énoncé qui fait porter "l'équivalence » sur le monde, et non sur les mots (ce qui sera montré plus loin) et qui, en outre, ne présente pas, avec synonyme, une relation d'équivalence, mais plutôt d'implication : on peut paraphraser ce ne doit pas être synonyme par/cela ne doit pas entraîner/.

Par ailleurs, et corrélativement, l'emploi de synonyme peut avoir des fonctions discursives diversifiées dans les énoncés : ainsi parfois, plutôt que de supposer une perception de «synonymie », l'énoncé l'impose. C'est le cas dans nombre de traités où le jugement de « synonymie » sert à l'auteur à présenter une équivalence de termes, utile pour la démonstration en cours ou ultérieure :

(2) L'être suprême doit être la suprême unité, puisque être et unité sont synonymes. (Fénelon, Traité de l'existence et des attributs de Dieu, 1713)

quitte à ce que cette équivalence n'en soit décidément plus une, et que l'énoncé, heurtant le sens commun, constitue un coup de force discursif en assumant le paradoxe - les mots ou expressions sont alors antonymes, ou en relation de contradiction :

(3) Il suit de là que tout encouragement au travail, toute récompense décernée à l'industrie, autre que le prix naturel du produit, est un don gratuit, un pot-de-vin prélevé sur le consommateur, et offert en son nom à un favori du pouvoir, en échange de zéro, de rien. Encourager l'industrie est donc synonyme au fond d' encourager la paresse : c'est une des formes de l'escroquerie. (Proudhon, Système des contradictions économiques ou Philosophie de la misère, 1846)

L'article présente les résultats d'un travail mené sur 832 énoncés issus de Frantext (à partir du $17^{\mathrm{e}}$ siècle), dans lesquels synonyme(s) (et dérivés) est le point d'entrée et la trace discursive d'une mise en relation, exprimée syntagmatiquement, entre un $\mathrm{X}$ et un $\mathrm{Y}$ (X est le synonyme de $Y$, X et $Y$ sont synonymes, par exemple). Cette recherche m'a permis de constater la diversité des fonctions discursives de synonyme (selon les périodes, selon les genres), et d'observer ses différentes valeurs sémantiques. Proposant dès à présent une observation générale, je soulignerai le fait (peut-être banal au demeurant) que synonyme et alii renvoient à une relation et, fait moins banal, que ces mots métalinguistiques renvoient davantage à une relation entre mots et monde (choses, expérience du monde) et surtout mots et notions qu'à une relation entre unités lexicales (entre signes linguistiques) - le signe linguistique n'étant cependant que rarement transparent, " traversé », purement dénominatif. En réalité, avec la relation exprimée entre $\mathrm{X}$ et $\mathrm{Y}$, les énoncés font apparaître une relation complexe et digne d'intérêt entre l'expression et ce qu'elle exprime, mêlant inextricablement sens et référence.

La première section exposera différentes valeurs de la synonymie dans les textes métalinguistiques (textes théoriques et dictionnaires). La deuxième section sera consacrée à la présentation du corpus (les corpus) et des angles d'attaque adoptés. J'exposerai dans une troisième partie des résultats quantitatifs et des analyses contextuelles.

(2) En ligne http ://amoureuxauban.net/. Je souligne dans les exemples les éléments X et Y entre lesquels est posée la « synonymie». 


\section{Relation de synonymie, et sens de synonyme-synonymie}

\subsection{Dans les textes théoriques}

J'entends par «textes théoriques » les ouvrages et articles à visée métalinguistique, produits par des rhétoriciens, grammairiens et linguistes. Ma présentation sera volontairement succincte, mais il me parait utile de rendre compte autant que possible de diverses conceptions théoriques de la notion de synonymie, dans la mesure où elles se reflètent parfois dans les énoncés de mon corpus, ou dans la mesure où, justement, ceux-ci s'en éloignent.

Pour les linguistes, il semble que, de manière consensuelle, la synonymie soit considérée comme une relation de sens (proximité, équivalence - un "même sens »), entre unités lexicales (UL désormais), ce qui la distingue de la relation de paraphrase. Le consensus apparaît également quand il s'agit de souligner qu'il n'existe pas d'identité totale de sens entre UL - pas de synonymes parfaits ; on trouve d'ailleurs déjà cette affirmation, non seulement chez Girard ${ }^{(3)}$ et, en 1730 , chez Dumarsais - qui affirme en conclusion de l'article S'il y a des mots synonymes : « S'il y avait des synonymes parfaits, il y aurait deux langues dans la même langue » (1988: 236) -, mais aussi antérieurement, chez les Anciens (grammairiens et rhétoriciens latins des premiers siècles de notre ère $\left.{ }^{(4)}\right)$. La constatation est exploitée par Bréal (2005: 43-49) avec le concept de « loi de répartition », repris par Ullmann (1959)... Tamba (2005) propose une analyse complémentaire en situant la différence entre synonymes dans la connotation - la dénotation étant identique. Dans tous les cas, la synonymie est distinctive et, explicitement ou implicitement, systémique - structurale, fût-ce avant la lettre. On sait d'ailleurs que l'exemple des synonymes craindre, redouter, avoir peur sert à Saussure (1985: 160) à illustrer la notion de valeur, corollaire de celle de système.

Est donc «structurale " cette conception de la synonymie - celle des linguistes, qu'on trouve également dans les dictionnaires de langue (c'est une des acceptions de synonymie et de synonyme dans tous les dictionnaires consultés : le Littré (1872), le TLF, le Dictionnaire de l'Académie (depuis la $4^{\mathrm{e}}$ édition de 1762), le Dictionnaire Historique Robert $(R H)$, etc.).

L'ensemble de ces conceptions font de la synonymie une relation de sens entre signes linguistiques, et non entre « choses » ou entre signes et « choses ».

Il en est autrement des acceptions anciennes de synonymie, dont le sens a évolué depuis son origine chez Aristote ${ }^{(5)}$, en passant par sa traduction en latin : Chevalier (1997) signale que la synonymie correspond chez les Anciens à des «noms différents pour une même chose (res) ». C'est aussi ce que décrit Desbordes (art. cit.). On renverra à sa description très détaillée des notions (entremêlées ou distinctes) d'homonymie et de synonymie chez différents auteurs, et surtout selon

(3) La Justesse de la Langue Françoise, ou les différentes significations des Mots qui passent pour Synonimes (1718), cité par Adamo «En marge d'une réédition de La Justesse de la langue françoise de l'Abbé Gabriel Girard (1718) » in Berlan F. et Bouverot D. (dir.) (2007), pp. 15-40.

(4) Voir Desbordes (1988:84-86).

(5) Chez lequel la relation est aussi entre noms : " noms dont le sens est lié (sun) par un genre commun, mais qui ont des sens différents (différences spécifiques : ainsi vert et rouge par rapport à couleur) » $(R H)$. Voir aussi la présentation des différentes « synonymies » chez Honeste «Entre ressemblance et différence : synonymie et cognition», in Berlan, F. et Bouverot, D. (dir.) (2007), pp. 160-174. 
différentes perspectives - dialectique, rhétorique et grammaticale. J'en retiens deux informations pertinentes pour le présent propos :

(i) la synonymie peut être chez les Anciens une relation entre dénominations d'une même chose ;

(ii) du point de vue de la rhétorique, il s'agit d'une pratique liée à la recherche d'ornement ${ }^{(6)}$. C'est, dans ce cas, une figure (d'amplification), « pratique médiévale, devenue systématique au XVI ${ }^{\mathrm{e}}$ siècle » qui consiste à « écrire par accumulation de synonymes (notamment de binômes synonymiques) ${ }^{(7)}$ " (Fuchs 1994 : 48) - cette acception rhétorique est signalée à synonymie dans le Littré, le $R H$ et le $T L F$. Par la suite, la figure devient gratuite et elle est mal perçue, considérée comme pléonasme et enflure ${ }^{(8)}$. Certains énoncés de Frantext témoignent de ce discrédit, particulièrement au $17^{\mathrm{e}}$ siècle :

(4) Si l'homme que vous connoissez, et qui fait toute sa gloire d'estre en vostre approbation, vouloit enfler ses escrits de ceux d'autruy; s'il vouloit à tout propos user de redites importunes; faire entrer par force dans ses discours de longues et ennuyeuses traductions : en un mot, monsieur, s'il vouloit desplier ses lieux communs ; je puis dire sans exagerer les choses, qu'il pourroit faire des livres de la taille de calepin. Mais son ambition non plus que la vostre, n'est pas de remplir les bibliotheques ; [...] Il fait assez d'autres pechez, sans aller grossir un volume de synonymes, d'amplifications, de digressions, qui seroient subjetes à correction ${ }^{(9)}$. (Guez de Balzac, Dissertations critiques, 1654)

Quant à la conception « dénominative » (i), elle correspond à une acception de synonymie mentionnée par le Littré :

$3^{\circ}$ En histoire naturelle, concordance de divers noms qui ont été donnés à un même animal, à une même plante. La nomenclature et la synonymie,

et c'est celle qui a cours dans les traités d'histoire naturelle, de sciences humaines ou de philosophie où les « synonymes » sont des noms, savants ou vulgaires, qui renvoient à une même chose. On trouve cette valeur dénominative de Buffon :

(5) Nomenclature des singes. Euvres deBuffon avec la synonymie et la classification de Cuvier augmentées des observations et suppléments des plus célèbres naturalistes mises en ordre et annotées par J. Pizzetta 500 gravures tome quatrieme. (1766)

\section{à Lévi-Strauss, par exemple :}

(6) Le terme statistique a une fonction logique, et pas seulement morale. Il renvoie au système de découpage adopté par l'auteur en question ou par un confrère, et permet donc au spécialiste d'opérer les transformations indispensables pour résoudre les problèmes de synonymie : de savoir, par exemple, que Juniperus occidentalis Hook est le même être que Juniperus utahensis Engelm.., alors que, sans le nom des inventeurs ou parrains, on pourrait conclure à deux êtres différents. ( $L a$ Pensée sauvage, 1962).

(6) Voir, à ce propos, les fines remarques de Quintilien, cité par Desbordes (pp. 84-85).

(7) Voir Buridant (1980) pour la description de l'usage de binômes synonymiques: deux mots synonymes sont adjoints, liés par un coordonnant. La visée est communicative et didactique : il s'agit d'expliciter par le procédé un sens présumé obscur, ou un emprunt.

(8) $C f$. Buridant (art. cit.). Dumarsais, sous l'entrée pléonasme, critique cette pratique (p. 322).

(9) Je maintiens la typographie et l'orthographe issues de Frantext. 
En résumé de ce qui précède, soulignons que, dans les écrits théoriques mêmes, la notion de synonymie connaît plusieurs valeurs. On peut s'attendre à ce qu'une telle diversité (et plus encore) se reflète dans les textes.

\subsection{Autres sens de synonyme-synonymie : témoignages lexicographiques}

On le voit, la conception « structurale » de la synonymie n'est qu'une valeur parmi d'autres... Comme indiqué plus haut, on trouve cette acception dans les articles de synonyme et synonymie des dictionnaires de langue, souvent en première place (sauf dans le $R H$, qui adopte un ordre historique et donc rapporte, en premier lieu, l'acception aristotélicienne). Mais d'autres acceptions interviennent ensuite ; je les mentionne rapidement, en cumulant les informations concernant les deux mots (si elles figurent, car l'entrée synonymie est moins développée).

Les acceptions qui apparaissent après l'acception « structurale » sont dites « figurées » : fig. (NPR), P. anal. (TLF), Par figure $(R H)$. Il s'agit grosso modo de la même acception (non mentionnée par les Dictionnaires de l'Académie ni par le Littré), que je cite à partir du TLF :

B. - P. anal. Synonyme de. Comparable, semblable à ; qui fait figure de symbole, de type. Boue est synonyme de honte (HUGO, Misér., t. 2, 1862, p. 549).

Enfin, il faut mentionner une dernière acception, celle de «titre d'ouvrage », qu'on trouve dans le Littré :

$4^{\circ} \mathrm{Au}$ plur. Titre de certains ouvrages, en forme de dictionnaire, dans lesquels la différence des mots synonymes est expliquée (il prend une majuscule). Les Synonymes latins de Gardin Dumesnil. Les Synonymes français de Girard. Les Synonymes de Lafaye.

Ces deux dernières valeurs - «symbole » et «titre d'ouvrage » - sont présentes dans mon corpus. Toutefois, la majorité des emplois rencontrés ne correspond à aucune de ces valeurs : j'ai signalé en introduction celle d'antonymie et celle d'implication ; on trouve également une relation associative (métonymique) ; d'autres me paraissent tout simplement inclassables.

\section{Corpus}

Les dictionnaires de langue portant témoignage des usages des mots, il n'est pas étonnant que les acceptions de synonyme et synonymie soient plurielles : les définitions sont une trace de la pratique, savante et non savante, de ces mots. Ici s'ajoute la spécificité de synonyme et alii, qui sont, on l'a dit, des mots métalinguistiques, et portent de manière privilégiée sur le sens : par là même, ils constituent un « témoignage épilinguistique », selon le terme de Bouverot (2007), qui étudie $s y$ nonyme dans les exemples du $T L F$; ils sont la marque d'une observation réflexive et d'une compétence sémantique de la langue ${ }^{(10)}$.

(10) Cf. aussi Julia (op. cit. p. 13). Je distingue les termes «épilinguistique » et «métalinguistique », en me basant sur les analyses éclairantes qu'en fait dans son introduction Auroux (1994), et que je résume ici : si l'activité épilinguistique peut se faire de manière plus ou moins consciente, « le véritable savoir est métalinguistique, c'est-à-dire représenté, cons- 
C'est du moins le point de départ de mes analyses. Comme on le verra, les énoncés observés illustrent également d'autres compétences : celle, par exemple, du témoin de son époque pour lequel l'évolution des mots et de leurs relations «synonymiques » reflètent l'évolution des mœurs et de la culture (de nos jours, X est devenu (le) synonyme de $Y$ ) ; celle de l'observateur de la pratique langagière d'autrui...

\subsection{Les corpus de textes : " corpus univers ", " corpus de travail »}

Recherchant une diversité d'époques et de contextes pour l'emploi de synonyme et dérivés, j'ai opté pour le recours à la base de données textuelle Frantext, qui présente l'avantage de cette (relative) diversité, et les facilités d'accès que l'on connaît - observons toutefois que ce choix n'est pas totalement satisfaisant, car Frantext est loin de refléter la diversité des pratiques langagières écrites : on n'y trouve par exemple pas de textes journalistiques et, pour l'essentiel, des genres « savants » et des auteurs « agréés ", toutes époques confondues. Une autre limite est celle de l'empan cotextuel proposé, souvent étroit pour les textes sous droits.

Je considère la base dans son ensemble comme un "corpus univers » ${ }^{(11)}$, au sein duquel j'ai sélectionné le corpus proprement dit, selon un critère simple : celui de la présence d'une occurrence au moins de synonyme ou de ses dérivés (j'ai éliminé les textes du $16^{\mathrm{e}}$ siècle, pour des raisons de compréhension). Le résultat de cette sélection constitue un ensemble de textes - c'est mon « corpus de travail ». La sélection n'est pas anodine : si le corpus univers correspond à une simple ressource, le relevé du corpus de travail (les textes « appelés » par l'emploi de synonyme) constitue déjà un lieu significatif d'interprétation, de par les genres qui s'y trouvent préférés.

On jugera de la portée de la sélection en comparant la répartition en genres du corpus univers (partie $1 \mathrm{du}$ tableau) et du corpus de travail (partie 2 ( $c f$. page suivante).

Comme on peut le voir en comparant les deux tableaux, la présence d'une occurrence au moins de synonyme ou de ses dérivés réduit drastiquement le nombre de textes sélectionnés. La répartition en genres change aussi considérablement. Pour ne relever que deux exemples, le genre traité-essai passe de $28 \%$ dans Frantext à $56 \%$ dans le corpus de travail, et le théâtre passe de $17 \%$ à $2 \%$. Le genre roman se maintient, principalement dans les textes des $20-21^{\text {e }}$ siècles, où il est très représenté : tous genres confondus, les textes de cette période correspondent à $44 \%$ du corpus univers et $54 \%$ du corpus de travail ; le genre roman y occupe $56 \%$.

En effectuant une comparaison entre genres au sein du tableau 2, on remarque notamment la prééminence du genre traité-essai : celle-ci me parait s'expliquer

truit et manipulé en tant que tel à l'aide d'un métalangage» (p. 23). Le mot synonyme et ses dérivés, les mots sens ou signifier également, sont bien des mots métalinguistiques, et c'est pourquoi je n'utiliserai que ce qualificatif. Il s'avère néanmoins que, dans les faits, comme je le montrerai, le mot métalinguistique synonymen'est pas réservé à des jugements métalinguistiques.

(11) Je reprends les termes que j'ai utilisés dans ma thèse (Lecolle, M., 2003, Métonymies et figures de référenciation dans la presse écrite généraliste. Analyse sémantique et rhétorique, thèse de doctorat, Université Toulouse-Le Mirail). L'essentiel est, avec ces termes, de distinguer ce qui correspond à une « ressource», une « archive» (le « corpus-univers ») et ce qu'on en extrait sur certains critères, différents selon l'étude (le « corpus de travail »). 
PARTIE 1

composition du corpus univers $(\text { Frantext })^{(12)}$
PARTIE 2

composition du corpus de travail ${ }^{(13)}$

\begin{tabular}{|l|c|c|c|c|}
\hline genres & $\begin{array}{c}\text { nombre } \\
\text { de textes }\end{array}$ & pourcentage & $\begin{array}{c}\text { nombre } \\
\text { de textes }\end{array}$ & pourcentage \\
\hline conte-récit & 2 & $0 \%$ & 1 & $0 \%$ \\
\hline correspondance & 157 & $4 \%$ & 24 & $6 \%$ \\
\hline éloquence & 48 & $1 \%$ & 1 & $0 \%$ \\
\hline essai-autobiographie & 4 & $0 \%$ & 4 & $1 \%$ \\
\hline mélanges & 7 & $0 \%$ & 4 & $1 \%$ \\
\hline mémoires & 175 & $5 \%$ & 24 & $6 \%$ \\
\hline mémoires-autobiographie & 39 & $1 \%$ & 9 & $2 \%$ \\
\hline pamphlet & 22 & $1 \%$ & 6 & $2 \%$ \\
\hline poésie & 482 & $12 \%$ & 8 & $2 \%$ \\
\hline récit de voyage & 55 & $2 \%$ & 3 & $1 \%$ \\
\hline roman & 1106 & $28 \%$ & 85 & $21 \%$ \\
\hline théâtre & 652 & $17 \%$ & 8 & $2 \%$ \\
\hline traité et essai & 1103 & $28 \%$ & 227 & $56 \%$ \\
\hline autres & 30 & $1 \%$ & & \\
\hline Total & $\mathbf{3 8 8 2}$ & & $\mathbf{4 0 4}$ & \\
\hline
\end{tabular}

par le fait que synonyme, et plus encore synonymie et synonymique (voir infra $\S 2.3$.), sont des mots « savants », utilisés à des fins réflexives ou descriptives de la langue et des langues, mais aussi de la pensée, de l'expérience, des mœurs ; des mots aussi qui ont souvent pour fonction de «fixer les idées » en fixant les termes (voir par exemple l'énoncé de Fénelon : être et unité sont synonymes) - toutes fonctions qui trouvent naturellement leur place dans des traités ou essais.

\subsection{Unité et diversité du corpus de travail}

À l'intérieur de cette unité que constitue simplement la présence de synonyme et dérivés, les textes sont divers de par leur époque et leur genre, mais également dans le cadre d'un même genre. Pour le genre traité-essai, les textes diffèrent principalement selon leur thématique. En voici quelques-unes : on trouve naturellement des essais et traités sur le langage, la langue ou le style (Bouhours, Fénelon, Marmontel, Destutt de Tracy, Diderot, Saussure, Hagège), mais aussi des essais de philosophie, des traités de logique $\left(17^{\mathrm{e}}\right.$ siècle $)$, d'histoire et de géographie $\left(19^{\mathrm{e}}\right.$ et

(12) Il s'agit de la composition en genres de l'ensemble de Frantext en juin 2008. J'ai opéré le rapprochement de certains genres (traité et essai), qui sont parfois distingués mais souvent conjoints dans la description des textes de la base. L'augmentation du nombre de textes dans Frantext pendant l'été 2008 ainsi que 1'enrichissement en précision des genres textuels par les administrateurs expliquent la présence de la case « autres » : je n'ai pas pu reconstituer la répartition de manière satisfaisante a posteriori (décembre 2008). Etant donné la taille limitée des corpus, les pourcentages sont présentés sans décimale et arrondis. C'est ce qui explique les $0 \%$ (chiffres en dessous de $0,5 \%$ ).

(13) À partir des informations documentaires des textes de Frantext, j'ai opéré la répartition en genres du corpus de travail (regroupements et simplifications), en reprenant les genres proposés dans la base. 
$20^{\mathrm{e}}$ siècles), de médecine et de mathématiques, ou encore portant sur l'alchimie et les alchimistes... On a aussi la surprise de rencontrer des textes comme Physiologie du gô̂t ou Méditations de gastronomie transcendante de Brillat-Savarin (1825), ou un article du Larousse Ménager (1926) portant sur les systèmes de chauffage centraux...

Certains auteurs sont plus représentés que d'autres (par exemple Michelet, Hugo, Proudhon, Bremond, Renan...), employant davantage que d'autres synonyme(s), et certains dans un style particulier, repérable à travers plusieurs occurrences. On retrouve ainsi 5 fois chez V. Hugo la structure que je note $X$ avoir un synonyme : $Y$, et dont voici deux exemples :

(7) Le Passé a un synonyme : l'Ignoré. (L'Homme qui rit, 1869)

(8) l'Humanité a un synonyme : Égalité (Choses vues, 1885).

\subsection{Les occurrences de synonyme et dérivés : «corpus-inventaire »}

Une fois extraits de Frantext, les énoncés sont consignés dans une base de données access : c'est mon «corpus-inventaire ». Celui-ci comprend 832 occurrences, réparties en 791 occurrences de synonyme (dont 7 de sinonyme, forme ancienne), singulier et pluriel, 37 occurrences de synonymie(s) et 4 de synonymique(s). Synonymie(s) et synonymique(s) sont présents majoritairement dans le genre traité-essai ( $83 \%$ des occurrences de ces formes).

Dans la base access, chaque occurrence de synonyme et dérivés est décrite dans son cotexte, selon les critères suivants (je résume ${ }^{(14)}$ ) :

1. morpho-syntaxiques et syntaxiques : synonyme $(s)$ adjectif ou nom ; structure du segment (GN) contenant l'occurrence de synonyme(s);

2. morpho-syntaxiques : catégorie grammaticale des $X$ et $Y$ ( $Z$ et autres, parfois) mis en relation, flexion de $\mathrm{X}$ et/ou $\mathrm{Y}$, changement éventuel de catégorie et/ou de nombre entre $\mathrm{X}$ et $\mathrm{Y}$; présence d'un nom propre $(\mathrm{Np})$ en $\mathrm{X}$ ou $\mathrm{Y}$, comme en (9) :

(9) Pour les moins jeunes, le mot cirque est synonyme de Grock, des Fratellini, des Clérans, de l'inoubliable Rastelli. Pour les aînés, le cirque c'était Foottit et Chocolat et la piste nautique du nouveau cirque. (Collectif, Histoire des spectacles, sous la dir. de Guy Dumur, 1965)

3. logico-sémantique : 1'emploi de synonyme(s) a une fonction «référentielle», comme en (4), rappelé ci-dessous, (7) et (8) :

(4) l'homme que vous connoissez [...] Il fait assez d'autres pechez, sans aller grossir un volume de synonymes, d'amplifications, de digressions, qui seroient subjetes à correction. (Guez de Balzac, Dissertations critiques, 1654)

ou «prédicative », comme en (1), (2), (3), rappelé ici, et (9) ci-dessus :

(3) Encourager l'industrie est donc synonyme au fond d' encourager la paresse : c'est une des formes de l'escroquerie. (Proudhon, Système des contradictions économiques ou Philosophie de la misère, 1846)

(14) La description est «allégée » pour synonymique(s) et synonymie(s) : tous les critères ne sont pas pertinents. 
4. énonciatifs : présence éventuelle de modalisateurs ou de marques de prise en charge spécifiant et réduisant la portée du jugement de "synonymie » : selon $\underline{\text { moi, }}$ X est synonyme de Y ou pour telle personnelépoque, X est synonyme de $Y$. Par contraste, on voit que les exemples cités de Hugo (7) et (8), de Proudhon (3) et de Fénelon (2) présentent la «synonymie » comme générale, voire absolue ;

5. sémantiques : sens de la relation dite de «synonymie » par le scripteur - /implication/ par exemple en (1); /contradiction/ (antonymie) en (3); /symbole/ en (9). Dans les cas (nombreux) où la « synonymie» posée me parait propre à l'auteur et au texte, je n'attribue aucune « étiquette " sémantique ;

6. discursifs : fonction discursive en contexte de l'occurrence. Les emplois discursifs de synonyme(s) obéissent parfois à un objectif didactique, ou encore argumentatif, par exemple, comme dans (3) rappelé ci-dessus.

\section{Ce que disent de la « synonymie » les énoncés}

Les critères consignés dans la base me paraissent, seuls ou croisés, apporter des éléments d'interprétation sur la manière dont on emploie synonyme(s), à propos de quoi on utilise ce mot, et, en définitive, sur les valeurs de la relation de « synonymie ». Dans ce qui suit, je tenterai d'expliciter mes conclusions en les appuyant à chaque étape sur les informations que je tire du corpus et des critères choisis.

\subsection{La « synonymie » : une relation}

Cette affirmation peut paraître tautologique, étant donné que la synonymie est définie par les linguistes comme une relation (sémantique). Elle me semble néanmoins utile, dans la mesure où les valeurs de la " synonymie » rencontrées dans mon investigation auraient pu la contredire ${ }^{(15)}$. On rappellera d'ailleurs que la conception de la synonymie comme figure de rhétorique ne met pas principalement l'accent sur ce point de vue relationnel et paradigmatique, mais sur une perspective syntagmatique - ce point de vue relationnel me parait alors présupposé.

Examinons à présent cette question à travers les résultats du corpus-inventaire. Je me baserai dans un premier temps sur les critères descriptifs 1 (critère syntaxique : fonction du GN contenant synonyme(s)) et 3 (critère logico-sémantique), concernant uniquement synonyme $(s)$. Selon le critère logico-sémantique, le GN contenant synonyme $(s)$ est évalué comme " prédicatif » $(616$ occ.) ou " référentiel » (157 occ.). Une autre sorte de structure (13 occ.) est dite « existentielle» - comme dans : il y a (peu) de pensées synonymes. La répartition des trois « fonctions » ainsi distinguées est la suivante : prédicative $78 \%$, référentielle $20 \%$, existentielle $2 \%{ }^{(16)}$.

L'enjeu est le suivant :

(i) synonyme (s), dans une fonction considérée comme prédicative (attribut par exemple), est nécessairement relationnel : les différentes structures, souvent avec copule, du type $X$ est (le) synonyme de $Y$ ou $X$ et $Y$ sont (termes, mots, expressions) synonymes, peuvent se ramener à /synonyme de/.

(ii) en revanche, lorsque synonyme(s) occupe une fonction reconnue comme ré-

(15) C'est bien le principe d'une analyse en corpus, avec ses éventuels revers, que je défends ici.

(16) Auxquels s'ajoutent 1\% d'énoncés parataxiques : synonyme(s) figure dans une énumération. 
férentielle (sujet ou COD), cette propriété relationnelle ne va pas de soi. Ainsi en (10), que j'examine pour le moment de ce seul point de vue, les synonymes comme sujet grammatical ne fait pas explicitement état d'une relation entre un $\mathrm{X}$ et un $\mathrm{Y}$ :

(10) Il n'est facile dans aucune langue de concilier l'harmonie avec les autres qualités du style ; et si l'on veut imaginer une langue qui peigne naturellement, il faut la supposer, non pas formée successivement et au gré du peuple, mais composée ensemble et de concert, par un métaphysicien comme Locke, un poëte comme Racine, et un grammairien comme Du Marsais. Alors on voit éclore une langue à-la-fois philosophique et poëtique, où l'analogie des termes avec les choses est sensible et constante, non-seulement dans les couleurs primitives, mais dans les nuances les plus délicates; de manière que les synonymes en sont gradués du rapide au lent, du fort au foible, du grave au léger, etc. (Marmontel, Poétique française, 1763).

Dans cet énoncé de spécialité portant sur la langue (on y remarque la référence à la synonymie différentielle), la qualité relationnelle de synonymes n'est ni explicitée ni même mentionnée, mais on peut dire qu'elle est présupposée : de fait, elle me parait contenue dans le sens nominal de synonymes - /mots qui ont entre eux une relation de synonymie/.

En comparaison, cette qualité relationnelle apparaît bel et bien dans des énoncés où synonyme est en «fonction » référentielle, mais où $\mathrm{X}$ et $\mathrm{Y}$ sont textuellement présents. C'est le cas de (11), où le synonyme est sujet grammatical :

(11) Malgré les mélanges qu'amène forcément le commerce, le synonyme de l'Angleterre, c'est l'exclusion. (Michelet, Sur les chemins de l'Europe, 1874).

ou encore de (7) où un synonyme est COD :

(7) Le Passé a un synonyme : l'Ignoré. (Hugo, L'Homme qui rit, 1869)

Quant aux énoncés « existentiels », du type de (12), ils me paraissent eux aussi présupposer une relation ${ }^{(17)}$ :

(12) il n'y a pas de synonymes. Il n'y a que des mots nécessaires, et le bon écrivain les connaît. (Renard, Journal : 1887-1910, 1910).

Je résumerai ce qui précède en disant que la «synonymie » exprimée dans les énoncés avec synonyme(s) est bien une relation, posée le plus souvent (énoncés à "fonction » prédicative, et énoncés « référentiels » qui présentent un $\mathrm{X}$ et un $\mathrm{Y}$ ), présupposée dans d'autres cas. Il me faut cependant nuancer le propos : relation présupposée ou posée, la différence n'est pas négligeable, et il faudrait aussi analyser les énoncés de ce point de vue ${ }^{(18)}$.

Enfin, les analyses du mot synonymie lui-même confirment, dans une certaine mesure, cette qualité relationnelle de la «synonymie ». En fait, les énoncés avec ce mot se répartissent grosso modo en deux catégories :

(17) La négation, tout à fait fréquente dans ce type d'énoncés, devrait être analysée pour ellemême. Quoi qu'il en soit, elle ne met pas en cause la présupposition.

(18) En particulier selon les différences entre siècles : la proportion d'occurrences d'emplois référentiels de synonyme(s) sans X et Y me parait plus importante au $17^{\mathrm{e}}$, ce qui, peut-être, confirmerait la prégnance d'une pratique rhétorique de la synonymie. Encore faudrait-il avoir davantage de textes de cette période. 
(i) l'une est réellement et explicitement relationnelle (la synonymie avec/entre/de, rapport/relation de synonymie) ;

(ii) une autre présente la synonymie comme un fait acquis, mais du point de son résultat - une liste (de synonymes). La question de la synonymie est alors souvent abordée tout autrement : on évoque à son propos un problème pour la communication (il est alors par exemple question de réduction/élimination des synonymie (s) dans un cadre de technique documentaire ${ }^{(19)}$ ), ou une mauvaise pratique scientifique liée à une mauvaise classification, comme ici chez Lamarck :

(13) En effet, le défaut de règles convenues, relatives à la formation des genres, des familles et des classes mêmes, exposant ces parties de l'art à toutes les variations de l'arbitraire, la nomenclature en éprouve une suite de mutations sans bornes. Jamais elle ne pourra être fixée tant que ce défaut subsistera; et la synonymie, déjà d'une étendue immense, s'accroîtra toujours, et deviendra de plus en plus incapable de réparer un pareil désordre qui annulle tous les avantages de la science. (Lamarck, Philosophie zoologique, 1809).

Cette catégorie d'occurrences (ii) renvoie à la valeur « dénominative » de la synonymie ; elle n'est alors plus explicitement relationnelle.

\subsection{Une relation, mais entre quels termes?}

Le critère 2 est destiné à relever les catégories grammaticales de $\mathrm{X}$ et $\mathrm{Y}$ - les termes mis en relation. Ce que je nomme «termes » ici peut correspondre à des UL ou à des expressions ( $\$ 3.2 .1$ ci-dessous), mais également à des groupes syntaxiques ( 3.2.2. ci-dessous) : on en trouve des exemples en (1), où X et Y sont des GN (en $\mathrm{X}$ : la volonté de gérer l'immigration, et de protéger les intérêts des français, repris anaphoriquement par ce) et en (3), où il s'agit de propositions infinitives (en X : encourager l'industrie). L'absence de flexion, et l'absence de déterminant pour les noms conduit à première vue à envisager le terme en mention (et non en usage). La présence adjointe des mots terme, mot, expression (le mot (de) X), ou celle de guillemets renforce ce jugement, le contexte étant toutefois déterminant.

\subsubsection{Catégories grammaticales des termes}

Concernant les UL simples (sans déterminant pour les noms), elles se répartissent de la manière suivante, pour les catégories les plus représentées (je ne mentionne que le terme $\mathrm{X})$ : noms dont Np (39\%), adjectifs $(6 \%)$, verbes $(4 \%)$, quelques prépositions et adverbes. Il faut mentionner aussi des expressions nominales $(5 \%)$, verbales $(1 \%)$ et adjectivales : il s'agit de locutions figées, ou de suites de mots non figées. Voici deux exemples d'expressions nominales :

(14) Le feu ne cessait de briller sur l'autel que lorsque la famille avait péri tout entière; foyer éteint, famille éteinte, étaient des expressions synonymes chez les anciens. (Fustel de Coulanges, La Cité antique, 1864).

(15) Ainsi que nous l'avons vu précédemment, une notion devient scientifique par cela seul qu'elle devient rationnelle. Le mot de médecine rationnelle serait donc synonyme de médecine scientifique, mais il n'est pas pour cela synonyme de médecine expérimentale (Bernard, Principes de médecine expérimentale, 1878)

(19) Coyaud, Introduction à l'étude des langages documentaires, 1966. 
En réalité, (15) peut être analysé à deux niveaux : à un premier niveau syntaxique, rationnelle, scientifique, expérimentale, adjectifs, peuvent être admis comme réellement (quasi-)synonymes. À un second niveau, celui où est réellement posé par Bernard le jugement de «synonymie », les expressions médecine rationnelle, médecine scientifique et médecine expérimentale, dans un cadre de langue de spécialité, fonctionnent bien comme des blocs, sans qu'on puisse pour autant parler d'expressions figées. Ce type d'exemple permet de voir que X et Y (ici les termes soulignés) n'ont pas besoin d'être des UL (c'est-à-dire des éléments lexicaux, simples ou complexes, stables) pour que soit posé un jugement de «synonymie » entre eux. Le cas présent illustre davantage d'ailleurs une " proposition » de synonymie - je parlerai plus loin de fonction discursive " démonstrative » de l'emploi de synonyme(s).

\subsubsection{Le cas des groupes nominaux, des noms, adjectifs et verbes fléchis}

Une part significative correspond à des GN (en X, 23\% ${ }^{(20)}$ ) - avec déterminant, donc (voir ci-dessus (1), (7), (8) et ici (16)) :

(16) Seulement, le rire est-il synonyme de la joie ? (Hugo, L'Homme qui rit, 1869).

La distinction entre nom et GN me parait importante : le GN, comme le Np, est en effet le niveau syntaxique de la désignation d'un référent. Dans les cas de GN, on tient donc un indice permettant de supposer que l'élément $X$ visé par le jugement de "synonymie » est peut-être autre chose que le signe linguistique luimême : on n'aurait pas affaire alors à un «pur » jugement métalinguistique sur des mots. Sur ce point, un raisonnement similaire peut être fait mutatis mutandis à propos des verbes ou adjectifs fléchis :

(17) Mais je voudrais bien que tu connûsse [sic] celle dont je parle ; tu lui dirais ton sentiment, et je suis sûre qu'il aurait du poids sur son esprit : il faut que je vous fasse faire connaissance : j'aime beaucoup cette jolie-personne, quoique très-assurée que j'ai peu de crédit sur son esprit ; car elle est passablement orgueilleuse, ou entêtée (ce qui, je crois, est synonyme) (Rétif De La Bretonne, Le Paysan perverti ou les Dangers de la ville, 1776)

Dans tous ces cas, le mot n'est pas employé en mention (comme on pourrait s'y attendre dans le cadre d'un jugement métalinguistique sur des mots) mais en usage ou, plus fréquemment, comme en (17), en modalisation autonymique ${ }^{(21)}$, c'est-àdire en usage et mention : d'abord employés comme qualificatifs appliqués à une personne, les adjectifs sont ensuite, par le biais du pronom anaphorique ce, commentés en tant que signes. Au-delà de cet exemple, on trouve souvent X ou Y introduit par mot, titre, terme, expression, alors même qu'il est fléchi (accordé, pour les adjectifs), ou qu'il s'agit d'une expression non stabilisée : il est donc bien alors question, à la fois, du signe et de ce qu'il désigne.

Pour revenir aux GN, ils servent parfois à une mise en relation entre « objets » (au sens large). Et c'est bien ce que présente (1), où, plutôt que de mots, l'énoncé parle d'expérience du monde - de l'implication entre un choix politique et son résultat présumé ; ou l'exemple (16), où est questionnée la mise en relation, sur des

(20) Il est fréquent aussi que $\mathrm{X}$ soit GN et Y nom, comme en (8) : l'Humanité/Égalité.

(21) Dans les termes de Authier-Revuz (2003). 
bases expériencielles, d'un symptôme (le rire) et d'un sentiment (la joie) ; ou encore (9), avec Np, où la «synonymie » correspond en fait à une relation de symbole basée sur des connaissances du monde (le cirque/les Fratellini).

L'exemple (18), où X et Y sont également des GN repris anaphoriquement par un pronom féminin pluriel (elles), permet d'illustrer un cas de figure non pas réellement similaire, mais comparable du point de vue qui m'occupe : le jugement de « synonymie » est porté sur des notions, et non sur des éléments lexicaux. On peut dire ici que la synonymie est « dénominative», c'est-à-dire que ce sont deux objets (ici deux notions) qui sont dits « synonymes » :

(18) La conscience du lié présuppose la conscience du liant et de son acte de liaison, la conscience d'objet présuppose la conscience de soi ou plutôt elles sont synonymes. (Merleau-Ponty, Phénoménologie de la perception, 1945).

Nuançons cependant : à partir du cas fréquent où X et/ou Y sont des GN, il est difficile de porter un jugement général - chaque énoncé est particulier. Mais ce qui est certain, et ceci à travers l'ensemble des énoncés, c'est que l'expression d'un jugement de synonymie «structurale» (i.e. différentielle et portant sur le sens des mots eux-mêmes) est plutôt l'exception que la règle (ce qui ne parait en définitive pas étonnant). Plus intéressante est l'observation de ce qui est mis en relation : avec l'emploi de synonyme(s), le scripteur entremêle sens linguistique et notion, expérience, concept (scientifique, philosophique). Les exemples suivants illustrent également ce fait - que la « synonymie »soit affirmée ou niée :

(19) pour les pères cappadociens, incompréhensible n'est pas synonyme d'inconnaissable. (Collectif, Dictionnaire de théologie catholique, 1920)

(20) Dans mon dictionnaire privé, enfance et mémoire sont synonymes. (Pontalis, L'amour des commencements, 1986).

En définitive l'emploi de synonyme(s) ne relève pas strictement d'une pratique métalinguistique, le jugement (apparent) sur les mots servant souvent, avec synonyme(s) comme d'ordinaire, peut-être, à parler d'autre chose, et notamment à cerner des sentiments, présenter des concepts, appuyer un argument ou illustrer un phénomène. C'est ce que j'aborde plus bas en parlant des «fonctions discursives » de l'emploi de synonyme et dérivés.

\subsection{Une relation, pour qui ?}

Avant d'aborder cette question, je mentionne rapidement un autre point, qui lui est sans doute lié : comme plusieurs exemples précédents le montraient déjà, la relation de «synonymie » est souvent modalisée (en (21) avec presque, en (16) par la forme interrogative), ou rapportée à un cadre de référence, temporel, spatial ou concernant une langue ou une communauté de locuteurs.

(21) Il était d'ailleurs trop petit encore pour attacher à la bonté tout son prix : dans le langage des enfants, bon et bête sont presque synonymes [...] (Rolland, JeanChristophe: L'Aube, 1904).

En résumé, la « synonymie » est dans ces cas, non pas générale et absolue, mais " pour quelqu'un » (pour moi, dans mon dictionnaire privé, dans le langage des enfants, pour les pères cappadociens, chez les anciens, chez les peuples barbares). À rebours, certains textes présentent la «synonymie»comme incontestable, ou universelle (nécessairement, de tous temps) : 
(22) [...] sentir est aussi la même chose que penser. Quand on donne à ces deux mots la signification la plus étendue qu'ils puissent recevoir, ils sont nécessairement et exactement synonymes ; car tous deux, ils comprennent généralement toutes nos perceptions quelconques. (Destutt De Tracy, Élémens d'idéologie. 3. Logique, 1805).

Après avoir dit que sentir et penser sont la même chose, l'auteur appuie une affirmation peut-être trop vague et peu technique par le recours à la "synonymie", elle-même appuyée par les adverbes : synonymes ainsi renforcé intervient alors comme un argument, d'autant plus fort qu'il s'agit d'un mot savant.

\subsection{Une relation, pour quoi ? : fonctions discursives de l'emploi de synonyme(s)}

Ce que je nomme « fonction discursive » répond à la question suivante : à quoi sert, en contexte, l'emploi de l'occurrence de synonyme ou dérivé ?

Comme discuté plus haut, rares sont les cas où l'emploi de synonyme reflète une réflexion strictement métalinguistique ; il est même fréquent que les énoncés ne présentent pas du tout ce caractère. J'ai isolé plusieurs fonctions discursives, que je répartis pour la présentation en trois groupes :

1. dans un premier groupe : les fonctions " métalinguistique ", " métadiscursive », "poétique » et « réflexive » sont présentes dans des énoncés où l'attention est portée sur le langage, la langue, le mode de parler, le style et les pratiques d'écriture (Poétique française de Marmontel par exemple). Voici un exemple d'énoncé à fonction "métadiscursive»:

(23) Les esprits médiocres ne trouvent point l'unique expression, et usent de synonymes. Les jeunes gens sont éblouis de l'éclat de l'antithèse, et s'en servent (La Bruyère, Les Caractères, 1696)

2. fonction « démonstrative »- illustrée notamment en (2), (3), (7), (8), (22) : l'emploi de synonyme (souvent dans des traités) sert à introduire des termes propres à l'auteur, pierres angulaires d'une argumentation. Le temps verbal employé est principalement le présent : être et unité / sentir et penser sont synonymes ;

3. fonctions « didactique » et « illustrative »-je reviens sur ces dernières.

Bien entendu, les fonctions ne sont nullement exclusives l'une de l'autre, et il est même fréquent, selon mon interprétation, que plusieurs soient présentes simultanément. En outre, elles sont parfois difficiles à délimiter l'une de 1'autre. Tentons, pour les deux dernières.

L'étiquette « didactique » est donnée aux occurrences de synonyme(s) où le mot sert à présenter des notions théoriques, étrangères dans le temps (passé), géographiquement et/ou provenant d'autres langues et cultures (essentiellement dans les traités et essais). On a, dans ces énoncés, en quelque sorte des définitions par synonymie (comme dans un dictionnaire); dans ces cas, l'attention portée aux mots liés par le jugement de «synonymie " sert à l'introduction d'une culture - voir (19) -, ou à une description historique, comme ici :

(24) Bourgeois et citadin étaient des termes synonymes, et [...] nous savons que les villes allemandes se sont formées autour de marchés permanents, ouverts par un 
seigneur sur un point de son domaine. La population qui venait se grouper autour de ces marchés, et qui devint la population urbaine, était donc presque exclusivement faite d'artisans et de marchands. Aussi les mots de forenses ou de mercatores servaient-ils indifféremment à désigner les habitants des villes, et le jus civile ou droit urbain est très souvent appelé jus fori ou droit du marché (Durkheim, De la division du travail social, 1893).

L'étiquette « illustrative » signale que l'emploi de synonyme(s) sert à illustrer des faits développés par ailleurs dans le texte : le comportement d'une personne, une culture, des mœurs, un « air du temps » ${ }^{(2)}$. La « synonymie » est alors l'indice d'autre chose. D'une manière générale, les énoncés de ce type jouent le rôle d'un exemple dans une description. J'analyse de la sorte (9), (21) ci-dessus, et (25), où la mention de "synonymie » est d'autant plus étonnante qu'elle énonce en fait une sorte de paradoxe, celui d'une équivalence nouvelle entre esprit faible et bon esprit:

(25) On aime mieux passer pour leste et dégagé que pour un honnête nigaud, et, du moment que l'on associe à la morale quelque idée de pesanteur d'esprit, c'est assez pour qu'on la tienne en suspicion. De là l'extrême rabais où est tombé le titre de bon esprit. Ce titre, qui devrait être le plus beau des éloges est devenu presque synonyme d'esprit faible, et est accordé avec une étrange libéralité (Renan, L'Avenir de la science, 1890)

Comme dans cet exemple, on trouve dans cette catégorie d'énoncés, ainsi que dans ceux à fonction "didactique ", des références au passé, et par là même à un changement, celui des mœurs par exemple : par le biais de structures telles que $X$ devenir synonyme de $Y$ ou $X$ cesser d'être synonyme de $Y$, les remarques portent sur l'évolution de la langue, certes, mais la langue comme trace, comme repère, des mœurs, de la culture et de leur évolution.

On peut résumer les spécificités de chacune des trois dernières « fonctions discursives » de la manière suivante : tandis que l'emploi « didactique » cherche à recenser une terminologie (de domaine, d'époque) pour avancer dans un exposé, l'emploi « illustratif » fait jouer aux éléments lexicaux et à leur relation « synonymique » le rôle d'un indice, d'une trace de quelque chose (qui dépasse les mots). Quant à l'emploi « démonstratif», il cherche davantage à poser (imposer) un sens qu'à en rendre compte.

\section{Conclusion : que reste-t-il de la synonymie?}

J'ai jusqu'à présent peu abordé la question sémantique : quel est le sens donné à la relation posée par l'emploi de synonyme? Du moins, je ne l'ai pas abordée de manière directe. Mais les développements précédents apportent des éléments de réponse.

La « synonymie » des énoncés analysés est une relation que je dirai de « contenu » (par opposition à « forme ») davantage que de sens (de signifié, au sens saussurien) : en effet, comme on l'a vu, les termes reliés peuvent effectivement l'être par leurs signifiés linguistiques, mais le sont souvent aussi par leur contenu référentiel (expérience du monde, notions, objets).

(22) Cette fonction a des affinités avec la «fonction métadiscursive »: le comportement discursif d'une personne ou d'un groupe est alors présenté comme «illustratif». 
Cette « synonymie » est souvent « pour quelqu'un », relative. Quant elle n'est pas présentée ainsi, elle l'est pourtant malgré tout : les énoncés « démonstratifs » en sont une illustration, qui imposent discursivement une vision absolue d'équivalence synonymique - vision que la communauté linguistique peut ne pas partager - silence et mort sont-ils « synonymes » pour d'autres que pour Leiris ? (La Règle du jeu : 1 : Biffures, 1948).

On peut cependant donner quelques éléments de réponse directe à la question sémantique, sans proposer de quantification :

- certains énoncés reflètent à proprement parler une synonymie, ou une parasynonymie : par exemple joye, plaisir, contentement, satisfaction, delectation, douceur, gayeté, estat paisible, tranquille, serain, seur, sans trouble, indolence, tranquillité (Bernier, Abrégé de la philosophie de Gassendi, 1684) - on peut d'ailleurs contester certains mots ;

- une bonne partie d'entre eux illustrent la valeur symbolique de la synonymie, telle que présentée dans la définition du TLF. C'est le cas en particulier des énoncés où $\mathrm{X}$ ou $\mathrm{Y}$ est un nom propre : Rancon, premier synonyme pour moi de la beauté et de l'injustice (Giraudoux, Sigfried et le Limousin, 1922);

- une partie non négligeable, non par le nombre, mais par son caractère impré$\mathrm{vu}$, énonce une contradiction : les « synonymes » $\mathrm{X}$ et $\mathrm{Y}$ sont alors en réalité des antonymes; le double paradoxe de cette relation et de son expression assumée constitue alors un mouvement discursif d'une force considérable ;

- on trouve enfin des énoncés où la «synonymie » est en fait une relation de causalité (ou, ce qui est équivalent d'implication), ou une relation plus lâche, « associative » (la relation est métonymique, ou indicielle, comme lorsque Hugo rapproche le rire de la joie).

Que reste-t-il alors de la synonymie?

Si cette relation, vue à travers les jugements métalinguistiques exprimés dans 832 énoncés, a finalement peu à voir avec la stricte définition adoptée par les linguistes, l'étude apporte en revanche de riches indications sur l'usage des mots et la perception sémantique, sur le rapport des usagers à la langue, à leur discours et à celui d'autrui, sur leur évaluation du rapport entre langue et culture... De la synonymie elle-même enfin, on peut dire qu'elle participe à toutes les fonctions du langage : c'est un instrument de dénomination, de communication et d'explicitation, un outil réflexif. 


\section{Bibliographie}

AurouX, S. (1994) : La révolution technologique de la grammatisation. Liège, Mardaga.

AUthIER-REVUZ, J. (2003) : « Le Fait autonymique : Langage, langue, discours. Quelques repères », in Authier-Revuz J., Doury, M. \& Reboul-Touré, S. (éds) Parler des mots : le fait autonymique en discours, Paris, Presses de la Sorbonne Nouvelle, pp. 67-96.

BERLAN, F. et BOUVEROT, D. (dir.) (2007) : Le français moderne, 1 : « La synonymie en questions. Échanges entre les époques ».

BRÉAL, M. ([1897] 2005) : Essais de sémantique (Science des significations), Limoges, Éditions Lambert-Lucas.

BURIDANT, C. (1980) : «Les binômes synonymiques. Esquisse d'une histoire des couples de synonymes du Moyen Âge au XVII ${ }^{\mathrm{e}}$ siècle », Bulletin du Centre d'analyse du discours 4, pp. 5-79.

Chevalier, J.-C. (1997) : «La synonymie dans les manuels pédagogiques du début de la Renaissance », Langages 128, pp. 8-24.

DESBORDES, F. (1988) : "Homonymie et synonymie d'après les textes théoriques latins », in Rosier I. (dir.), L’ambiguïté. Cinq études historiques réunies par Irène Rosier, pp. 51-102.

DumARSAIS ([1730] 1988): Des tropes ou des différents sens. Présentation, notes et traduction F. Douay-Soublin. Paris, Flammarion, collection Critiques.

FUCHS, C. (1994) : Énonciation et paraphrase, Paris/Gap, Ophrys.

Glatigny, M. (1980) : «Contribution à la préhistoire de l'analyse sémique : le dictionnaire des synonymes de Guizot (1809) ». Bulletin du Centre d'analyse du discours 4, pp. 181-236.

JULiA, C. (2001), Fixer le sens? La sémantique spontanée des gloses de spécification du sens, Paris, Presses de la Sorbonne Nouvelle.

REY-DEBOVE, J. (1997) : «La synonymie ou les échanges de signes comme fondement de la sémantique », Langages 128, pp. 91-104.

SAUssure, F. de ([1972] 1985) : Cours de linguistique générale, Paris, Éditions Payot.

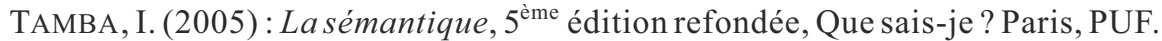

Ullmann, S. ([1952] 1959) : Précis de sémantique française, Berne, Éditions A. Francke S.A.

\section{Dictionnaires}

A TILF, CNRS : Académie Française. Dictionnaire de l'Académie française, quatrième édition (1762) : [en ligne] http ://atilf.atilf.fr/academie4.htm

— Dictionnaire de l'Académie française, huitième édition (1932-1935) : [en ligne] http ://atilf.atilf.fr/academie.htm

- Le Trésor de la Langue Française Informatisé (TLFi). [en ligne] http ://atilf.atilf.fr/tlf.htm

LE LITTRÉ, Dictionnaire de la langue française 1872. CD-Rom Texte Intégral. Marsanne, Redon.

Rey, A. \& Rey-Debove, J. (dir.) (1994) : Le Nouveau Petit Robert, Paris, Dictionnaire le Robert (NPR dans le texte).

REY, A. (dir) (2000) : Dictionnaire Historique de la langue française, 3 tomes. Paris, Dictionnaires le Robert $(R H)$. 